\title{
EXPERIMENT ON INITIATION MECHANISM OF DEBRIS FLOW: COLLAPSE OF NATURAL DAM IN A STEEP SLOPE CHANNEL
}

\author{
K.R.PATHAK ${ }^{1}$, Koichi SUZUKI ${ }^{2}$, Akihiro KADOTA ${ }^{3}$ and K.MATSUDA ${ }^{4}$ \\ ${ }^{1,4}$ Student member of JSCE, Graduate Student, Dept. of Civil and Environmental Engineering, Ehime University \\ (3 Bunkyo-cho, Matsuyama 790-8577, Japan) \\ ${ }^{2}$ Member of JSCE, Dr. of Eng., Professor, Dept. of Civil and Environmental Engineering, Ehime University \\ (3 Bunkyo-cho, Matsuyama 790-8577, Japan) \\ ${ }^{3}$ Member of JSCE, Dr of Eng., Research Associate, Dept. of Civil and Environmental Engineering, Ehime University \\ (3 Bunkyo-cho, Matsuyama 790-8577, Japan)
}

Formation of natural dams is a common process in gorges of the flow route of relatively steep gradient mountain rivers. A tremendous part of the flow accumulates upstream once such a dam forms in the way of regular flow. Consequently, the accumulated water submerges a large area causing inundation upstream and posing the threat of debris flow and flooding downstream. Once the dam is unable to hold the huge amount of amassed water destruction of the dam begins and brings debris flow disaster into the downstream areas, creating great havoc.

The present study attempts to discuss the experimental observation of different types of failure in a dam, e.g., slope failure at the frontal part and bed load transportation and debris flow at the body part by making trapezoidal shape of a natural dam. Experiments have been conducted using almost uniform-sized gravel. The collapse mechanism and its progress in the upstream direction show the trends of collapse in a progressive fashion, starting from downstream and advancing upstream.

\section{Key words: Debris flow, Mountain river, and Natural dam}

\section{INTRODUCTION}

Debris flow is a mass wasting process in which an ample amount of water is supplied to saturate the voids among the particles and is mixed with the mass of earth and stone. Debris flow, slope failures or shallow landslides occur somewhere in the world almost every year due to heavy rainfall or earthquake and even newly erupted volcanic ash that is deposited in the slope of volcanoes can cause debris flow easily with a little rainfall ${ }^{1)}$.

The initiation of flow has been related to the following three reasons. Firstly, landslide that turns into debris; secondly, destruction of a naturally built dam turning into debris flow, and lastly, surface water flow on a gully bed during heavy rainfall which mobilizes the accumulated mass ${ }^{2}$.

Natural dams are formed across the steep-slope channel of mountainous areas, mainly because of landslides, i.e., collapse of a bank etc. Forces of flowing water in and over the dam and a pressure of detained mass of water cause the movement and destruction of dam, and debris flow is likely to occur in downstream areas of the channel. The initiation mechanism of destruction of natural dams varies with the characteristics of dam forming materi- als, and channel as well as with the amount of past discharge.

Knowledge of debris flow is based largely on the contribution of Bagnold (1954) ${ }^{3)}$ and Takahashi $(1978)^{4}$. Many researchers have done almost similar studies regarding the failure mechanism of a natural dam and mobilization of channel bed using specific shape of the dam and the flow conditions ${ }^{5) 6}$. This study attempts to discuss different types of failure mechanism in a dam, e.g., slope failure at the frontal part and bed load transportation and debris flow at the body making use of trapezoidal shape of a natural dam and considering size of dam forming materials and discharge as parameters.

\section{EXPERIMENTS}

\section{(1) Set-up and procedure}

The experiments were carried out in an indoor laboratory flume of $15 \mathrm{~cm}$ in width, $30 \mathrm{~cm}$ in height and $10 \mathrm{~m}$ in length. The flume was equipped with adjustable bed slope and transparent sidewall at both sides. All the experiments of the present study were conducted at the slope of $1 / 5.57$. A dam $(15 \mathrm{~cm}$ wide, $10 \mathrm{~cm}$ deep and $1 \mathrm{~m}$ long) was made of sand or 


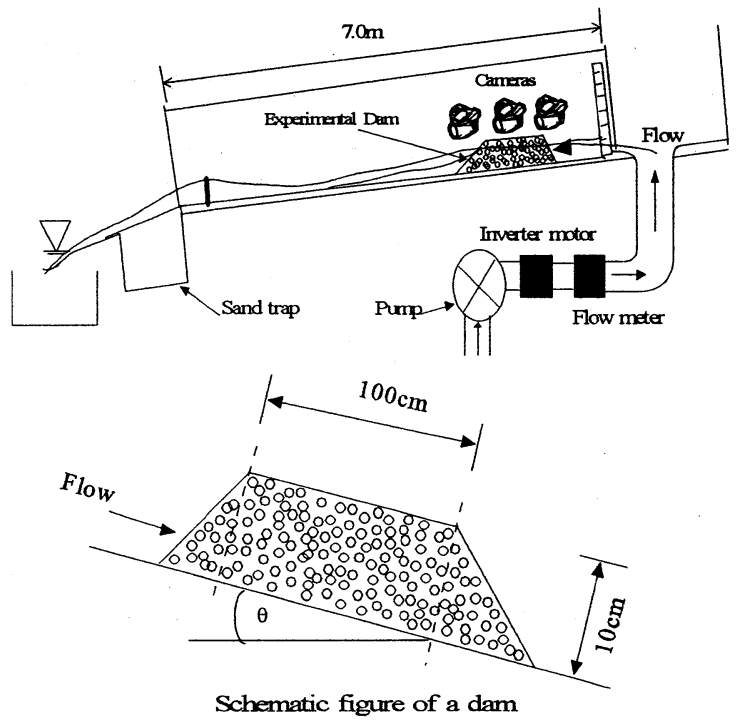

Fig.1 Schematic of experimental set-up gravel with almost uniform grain size of $1,4,7$, and $15 \mathrm{~mm}$.

Bed slope of the frontal part of dam was set almost equal to the internal angle of friction of the bed materials. This angle is basically a function of grading and angularity in the case of non-cohesive materials ${ }^{7)}$.

Experiments were conducted separately, changing the flow rate of water five times for each grain size. Consequently, altogether twenty cases were carried out. Water was lifted by a pump from the ground pool and collected in a chamber, located about $3 \mathrm{~m}$ upstream of the dam body, at the head of

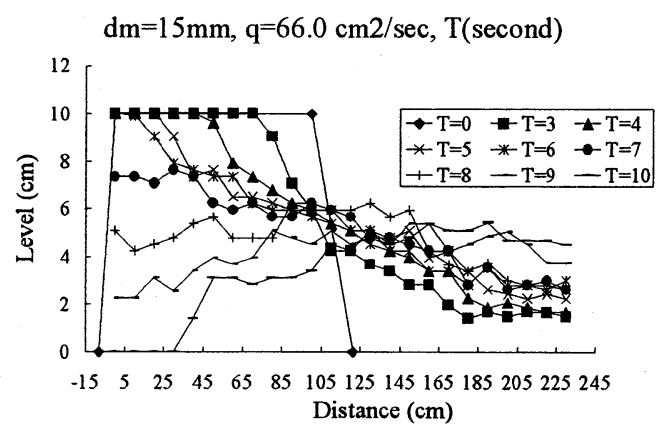

(a-1)
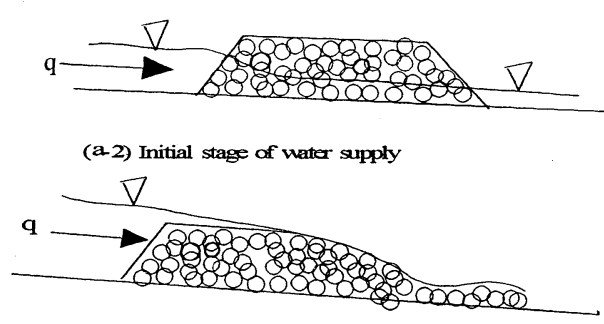

(a-3) Begining of collapse under constant discharge (a) GRAVEL the flume.

Discharges were supplied from the upstream end of the channel, where a dam was made for the observation of initiation mechanism of its destruction. A constant discharge was supplied until the destruction of dam out of its original position was completed and the rate of flow was reckoned manually using a measuring bucket and a stopwatch, in each case. Three video cameras were set at different locations to record continuous and simultaneous measurements of initiation process of debris flow. The schematic figure of the experimental set-up is shown in Fig. 1.

\section{(2) Experimental conditions}

Experiments were carried out in four runs and each run was conducted at a channel slope of $1 / 5.57$ with five different amounts of discharge. Four kinds of mean diameters of almost uniform gravel or sand were used to form the experimental dam. Experimental conditions are given in table 1 .

Table 1 Experimental conditions

\begin{tabular}{|c|c|c|c|c|c|c|}
\hline $\begin{array}{c}\text { Run } \\
\text { No. }\end{array}$ & $\begin{array}{c}\text { Grain size } \\
\text { Dm (mm) }\end{array}$ & \multicolumn{5}{|c|}{ Discharge $\left(\mathrm{cm}^{2} / \mathrm{sec}\right)$} \\
\hline Run1 & 1 & 20.67 & 26.00 & 34.67 & 64.00 & 84.67 \\
\hline Run2 & 4 & 18.00 & 44.00 & 53.33 & 65.33 & 76.00 \\
\hline Run3 & 7 & 26.00 & 44.00 & 58.67 & 62.00 & 98.00 \\
\hline Run4 & 15 & 32.67 & 33.33 & 66.00 & 83.33 & 101.33 \\
\hline
\end{tabular}

$\mathrm{dm}=1 \mathrm{~mm}, \mathrm{q}=64.0 \mathrm{~cm}^{2} / \mathrm{sec}, \mathrm{T}$ (second)

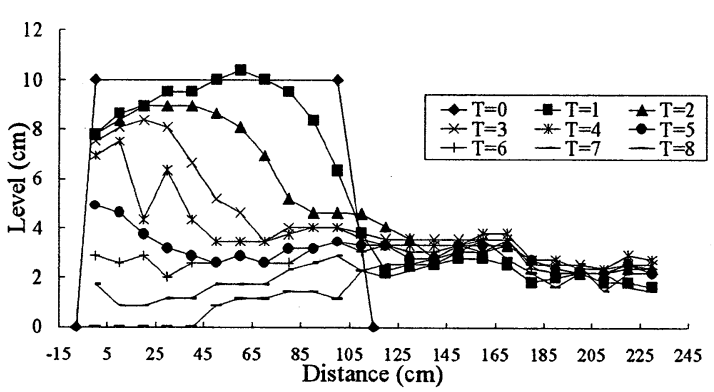

(b-1)
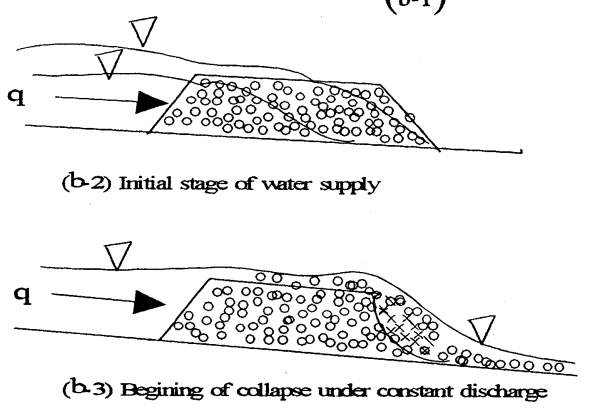

(b) SAND

Fig.2. Actual and schematic diagrams of initiation of collapse phenomena with the size of dam forming materials 


\section{RESULTS AND DISCUSSION}

\section{(1) Types of collapse}

Observation during the experiments revealed that the grain size of dam forming material is one of the crucial parameters governing the initiation mechanism of debris flow in the destruction process of the natural dam.

Schematic diagrams of Fig.2 show how the collapse of the dam proceeds with the grain size of dam forming materials. Graphs (a-1) and (b-1) show the actual process of destruction phenomena of experiments for gravel and sand respectively. In the case of smaller particles, flow proceeds as shown in

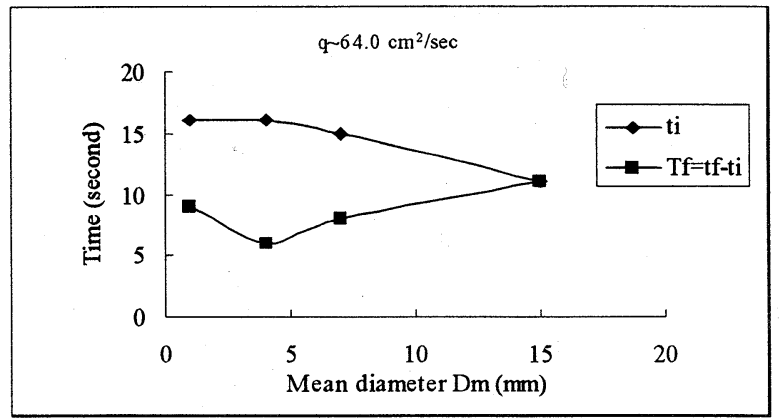

Fig. 4 Mean diameter versus time required for collapse

\section{(2) Time of complete collapse $t_{f}$}

In this experimental study, the time ' $t_{i}$ ' is defined by the time period between arrival time of flow at

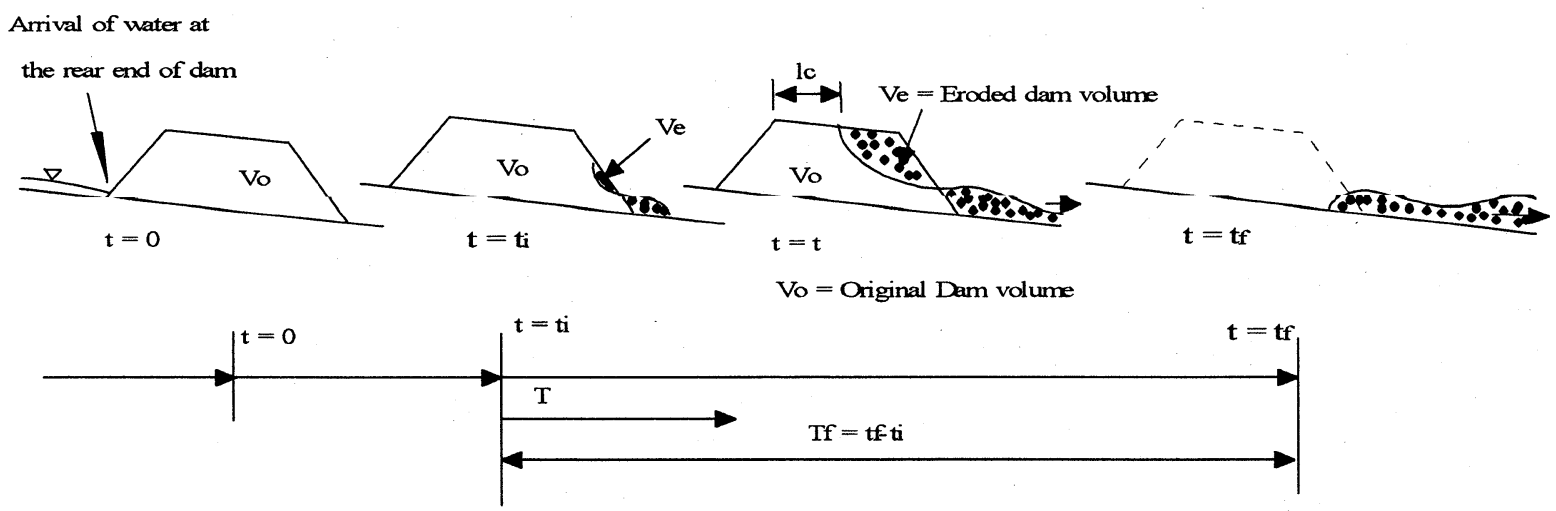

Fig.3 Definition sketch of time, eroded volume and collapse position $l_{c}$

diagram (b-2) and eventually collapse results as diagram (b-3). A portion of flow enters into the dam body itself and the remaining part accumulates and sets the upstream water level rising. Surface flow over the dam results as the water level surpasses the height of dam. Seepage and over toped flow advances in the downstream direction. As overtopping of flow begins, it erodes particles from the dam surface and a part of downstream face collapses initiating the destruction of the dam and continues as shown in (b-1). In the case of larger particles, a considerable part of the flow percolates through dam body itself as shown in (a-2). The rate of percolation was faster and greater than the setting of the water level upstream of the dam. The initiation of destruction started at the toe of the dam where the seepage water emerges on the surface as shown in (a-3). Examples of experimental results are depicted in the graphical plots in (a-1) and (b-1) of Fig.2. Initial destruction was begun near the downstream face of the dam and proceeded rapidly and progressively towards the upstream direction in the case of the dam made of larger-sized particles. Progressive failure seems to proceed in the upstream direction. Complete destruction was rather faster in the case of small-sized particles. the rear end of dam (where $t=0$ ) and the beginning of erosion near the downstream face of the natural dam. The time ' $T$ ' is the time period elapsed from the beginning of erosion. Similarly, ' $\mathrm{T}_{\mathrm{f}}$ ' is the time of complete collapse out of its original position of the dam. Fig. 3 diagrammatically depicts the definition of different terms of time, volume and collapse position.

Fig.4 shows the relationship between the grain size of bed materials and time required for the initiation of destruction and complete wash out of a dam from the original position. From the figure, it can be seen that initiating time of destruction of a dam decreases gradually as the size of the dam

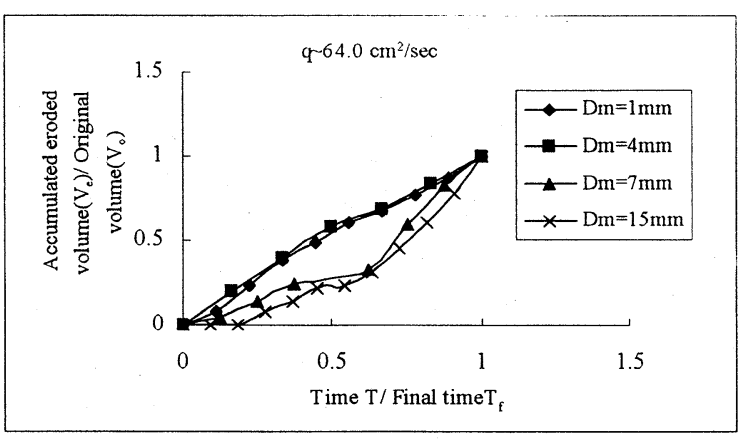

Fig.5 Relationship between non-dimensional eroded volume versus time at approximately same discharge 

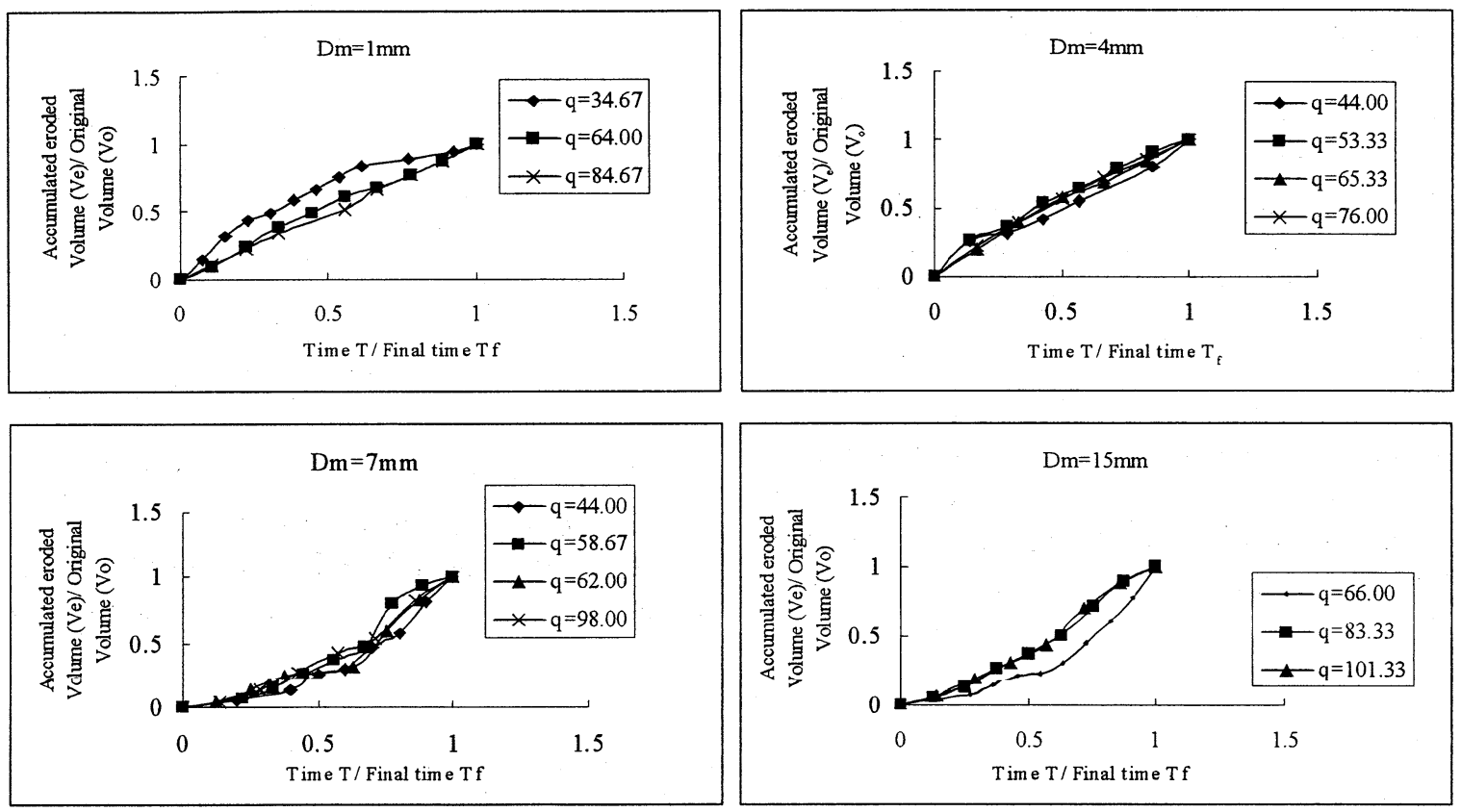

Fig.6. Relationship between non-dimensional time and eroded volume of various sizes of dam forming materials with alternation of discharge
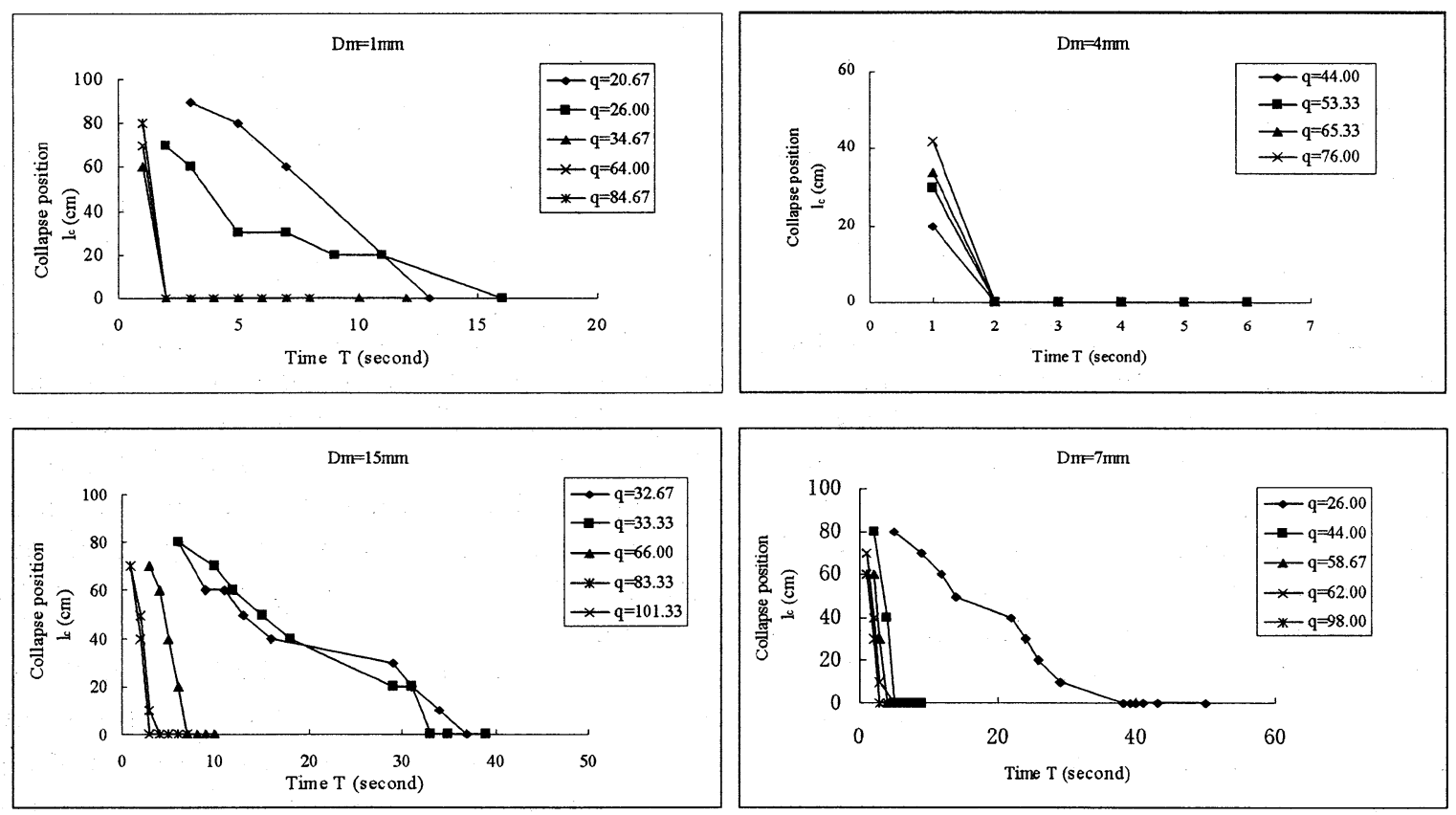

Fig.7. Advancement of collapse position with the discharge increment
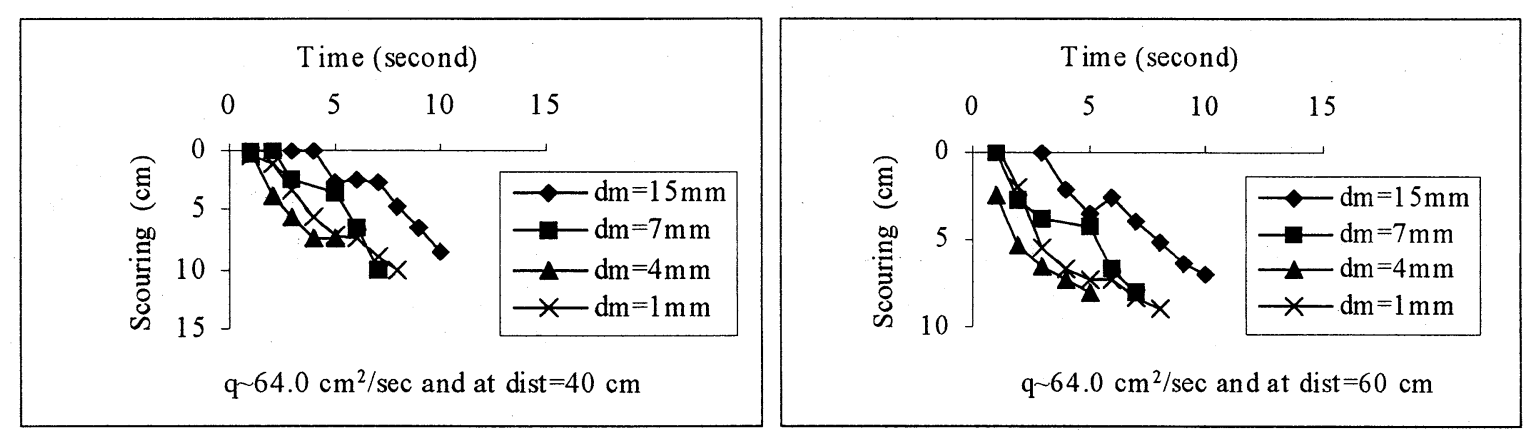

Fig. 8 Scouring at particular locations of dam with time 
forming materials increases.

The reasons might be poor permeability and higher waterholding capacity of smaller particles compare to larger ones. But, the time taken for the completion of collapse once destruction starts is longer for larger particles than smaller ones. This means the dam formed by small-sized particles collapses faster than the dam made of larger sized particles.

\section{(3) Temporal variation of eroded volume}

The eroded volume $\left(\mathrm{V}_{\mathrm{e}}\right)$ is defined by the volume eroded (accumulated) and transported from the original dam volume $\left(\mathrm{V}_{0}\right)$ at a time concerned. Fig.5 shows the agreement with the experimental observation made during the intiation process of debris flow with different grain sizes under almost the same discharge. The eroded volume at the onset of destruction is larger in the case of smaller particles and keeps advancing almost steadily. However, in the event of large-sized particles, the rate of eroded volume is smaller at the beginning of destruction and increases as time goes by.

This implies that in the case of small-sized particles, surface erosion at the body part and collapse at the frontal part of the dam work together to erode a greater volume than that of large-sized particles at the onset of debris flow. This may be due to a large differential pressure (pressure difference between saturated part and unsaturated part) and almost critical bed slope of the frontal part (bed slope of the frontal part in almost equal to the internal angle of friction). As time passes bed slope of frontal part becomes milder and modes of failure changes into debris flow from slope failure.

This phenomenon shows the importance of permeability and waterholding capacity of the dam forming material for the initiation mechanism of debris flow.

Fig. 6 shows how the destruction proceeds to collapse of the dam with the variation of the flow of water, i.e., discharge. Once enough discharge is supplied to initiate the debris flow for a particular size of material, there is no noticeable difference in the destruction process (in terms of eroded volume). In the case of smaller particles, destruction is linear. On the other hand, in the case of larger particles destruction rate is low at the beginning and increases as time passes.

\section{(4) Advancement of collapse position}

Fig. 7 shows the advancement of collapse position measured from upstream in the downstream direction. As is evident from the figure, the advancement of collapse is slow and destruction phenomena pro-
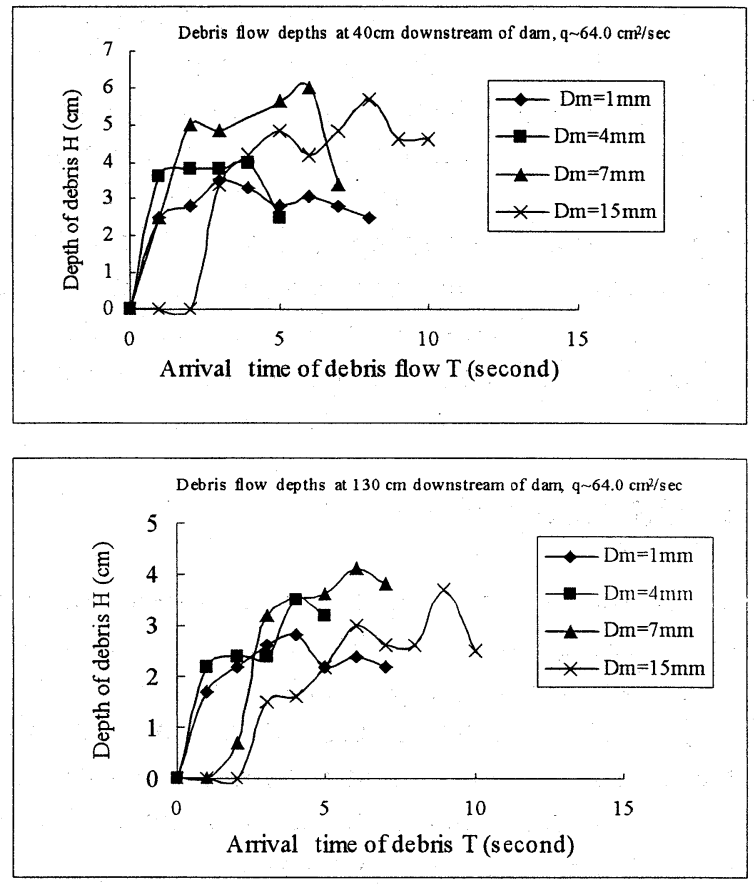

Fig.9. Debris flow depths downstream of the original dam body at $40 \mathrm{~cm}$ and $130 \mathrm{~cm}$ downstream

ceeds slowly at lower discharges. It is fair to infer that these small discharges are not enough to produce the debris flow. However, at greater discharges collapse occurs in a short period of time as seen in plots.

\section{(5) Temporal scouring}

Fig. 8 shows the temporal change in bed level at particular locations with the supply of approximately the same discharge for different mean diameters of bed material. To scour a given depth, time required for scouring is longer for larger sized material. However, the smallest sized material ( $1 \mathrm{~mm}$ in this study) shows a different tendency than larger sized. It took more time to scour a given depth than did the grain size of $4 \mathrm{~mm}$. This may be because of cohesive forces existing in this smallsized material, which is one of the important parameters to be considered for the analysis of initiation mechanism of debris flow.

\section{(6) Depth of debris flow downstream of dam}

Fig. 9 shows the debris flow depth at $40 \mathrm{~cm}$ and $130 \mathrm{~cm}$ downstream of the original position of the dam. Occurrence time of peaks is shorter for smaller particles than larger ones. Peaks of debris flow appeared after a time lag, which led to formation of multiple peaks. Heights of peaks of debris flow are higher in the case of larger particles than smaller ones. The implication of this could be partial destruction of the dam. Partial destruction was observed during the experiments. Water withholding capacity behind the dam decreases as the size of the 
dam decreases because of subsequent erosion and collapse. Partial remains of the dam could bear some pressure of water until it reaches a critical level. Once the exerted pressure of the accumulated water is more than a critical value, the dam again collapses abruptly showing a peak of flow just a moment after the previous peak. The same process repeats again and again until the dam materials wash out completely. This process causes the eroded volume of the dam to increase intermittently.

\section{CONCLUSION}

As for the larger particles, some parts of the discharge seepage through the dam body creating a slippage and collapse proceeded in the upstream direction. The quantity of eroded volume is smaller at the beginning and increases as time goes by. The nature of destruction of the dam made of largersized particles is rather progressive.

On the other hand, in dams made of smaller particles the destruction process is like a slope failure at the frontal part and bed erosion or debris flow like at the body portion of the dam.

The destruction process of a natural dam is a complex and seems to be governed by many factors. Among them supplied discharge, depth of flow, characteristics of dam forming materials, i.e. permeability, size, cohesion, angle of repose of materials, and so on seem the important factors to be considered in analyzing the phenomena. Furthermore, to generalize the results that have been obtained in this simple experiment theoretically, a rigorous analysis should be performed in future research considering the important parameters.

\section{APPENDIX-NOTATION}

$$
\begin{aligned}
\mathrm{Dm}= & \text { Mean diameter of grains used to form a } \\
& \text { dam }(\mathrm{mm}) \\
\mathrm{H}= & \text { Depth of debris flow }(\mathrm{cm}) \\
\mathrm{q}= & \text { Water discharge per unit width }\left(\mathrm{cm}^{2} / \mathrm{sec}\right) \\
\mathrm{T}= & \text { Time, measured from initiation of destruct } \\
& \text { ion of dam (second) i.e } \mathrm{T}=\mathrm{t}-\mathrm{t}_{\mathrm{i}} \\
\mathrm{t}= & \text { Time measured from the arrival }(\mathrm{t}=0) \\
& \text { of flow at the rear end of dam }(\text { second }) \\
\mathrm{t}_{\mathrm{i}} & \text { Time period between arrival time of flow } \\
& \text { at the rear end of dam and beginning of } \\
& \text { the erosion near the downstream face of } \\
& \text { the dam (second) } \\
\mathrm{t}_{\mathrm{f}}= & \text { Time required for complete wash out } \\
& \text { of a dam from its original position } \\
\mathrm{T}_{\mathrm{f}}= & \mathrm{t}_{\mathrm{f}}-\mathrm{t}_{\mathrm{i}}(\text { second) } \\
\mathrm{V}_{0}= & \text { Original volume of a natural dam }\left(\mathrm{cm}^{3}\right) \\
\mathrm{V}_{\mathrm{e}}= & \text { Accumulated eroded volume }\left(\mathrm{cm}^{3}\right)
\end{aligned}
$$

\section{REFERENCES}

1) Mizuyama, Takahisa.: Some Research Topics on DebrisFlow and Shallow landslide, $2^{\text {nd }}$ IAHR Symposium on River, Coastal and Estuarine Morphodynamics, 2001.

2) Takahashi, T.: Debris Flow, IAHR Monograph Series, Balkema, 1991.

3) Bagnold, R.A.,: Experiment on a Gravity-free Dispersion of Large Solids in a Newtonian Fluid Under the Shear, Proc. Royal Society of London, Series A, 225, 1954.

4) Takahash, T.,: Mehanical Characteristic of Debris Flow, J. Hydraulic Division, ASCE, 104, 1978.

5) Takahash, T., and Yagi.H.: Prediction of Debris Flow Discharge, Manuals, Disaster Prevention Research Institute, Kyoto University, No:26 B-2, 1983.

6) Tognacca, C., and Bezzola, G.R.: Debris Flow Initiation by Channel Bed Failure, Proceedings of First International Conference on Debris-Flow Hazards Mitigation: Mechanics, Prediction and Assessment, 1997.

7) Lambe, T.W., and Whitman R.V.: Soil Mechanics, SI Version, Series in Soil Mechanics, John Wiley and Sons, 1979.

(Received September 30, 2002) 